\title{
Anesthetic Care for a Pediatric Patient With Anti-N-Methyl- D-Aspartate Receptor Encephalitis
}

\author{
Asad A. Khawaja a, b, d , Mohammed Hakim ${ }^{\mathrm{b}}$, Joshua Uffman ${ }^{\mathrm{b}, \mathrm{c}}$, \\ Joseph D. Tobias ${ }^{b, c}$
}

\begin{abstract}
First characterized in 2007, anti-N-methyl-D-aspartate (NMDA) receptor encephalitis is an autoimmune disorder with the production of antibodies against the NMDA-type glutamate receptor. The process results in a constellation of psychiatric signs and symptoms with disorders of motor and respiratory function. We describe the anesthetic management of a patient with anti-NMDA receptor encephalitis who required anesthetic care during radiological imaging. Primary perioperative concerns include the potential for arrhythmias, hemodynamic compromise, respiratory failure, and central nervous system involvement including seizures, altered mental status, and psychiatric manifestations.
\end{abstract}

Keywords: NMDA; Encephalitis; General anesthesia

\section{Introduction}

Anti-N-methyl-D-aspartate (NMDA) receptor encephalitis is an autoimmune disorder in which antibodies are formed against the NMDA-type glutamate. The disorder was first reported in 2005 in a cohort of four young women who presented with psychiatry symptoms, altered mental status, hypoventilation, and memory deficits with coincidental ovarian teratomas [1]. It is postulated that the pathogenesis is the result of the effects of autoantibodies against NMDA receptors throughout the body. These auto-antibodies are formed in response to the neuronal tissue in the ovarian teratomas. The disorder has been most commonly reported in young African-American women

Manuscript submitted May 8, 2019, accepted May 30, 2019

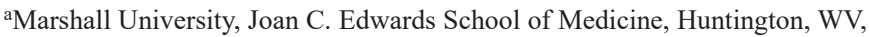
USA

bDepartment of Anesthesiology and Pain Medicine, Nationwide Children's Hospital, Columbus, OH, USA

'Department of Anesthesiology and Pain Medicine, The Ohio State University, Columbus, OH, USA

${ }^{d}$ Corresponding Author: Asad A. Khawaja, Marshall University Joan C. Edwards School of Medicine, 1600 Medical Center Drive, Huntington, WV 25701, USA. Email: khawaja2@live.marshall.edu

doi: https://doi.org/10.14740/jmc3310 between 18 and 35 years of age [2]. It is the second most common cause of immune-mediated encephalitis, after acute disseminated encephalomyelitis [3].

Patients typically evolve into a state of unresponsiveness with disturbances in motor and respiratory function after an initial presentation with psychosis, memory deficits, seizures, and language disintegration [4]. Prior to the identification of the exact pathogenesis of this immune-mediated process, many of these patients were erroneously diagnosed with schizophrenia and other mental health issues. The diagnosis is suggested by the clinical symptomatology, cerebrospinal fluid (CSF) lymphocytosis, and delta or theta activity on the electroencephalogram (EEG). Confirmation of the diagnosis is now possible by the demonstration of specific antibodies in the CSF against the NMDA receptor [4]. Treatment typically involves plasmapheresis, intravenous immunoglobulin therapy, as well as chemotherapy and surgery if a tumor is present.

Given the associated changes in central nervous system function and its association with abdominal teratomas, anesthesia is frequently required for radiological imaging or surgery. We present an adolescent with anti-NMDA receptor encephalitis who required anesthetic care for magnetic resonance imaging (MRI) imaging. End-organ involvement and its impact on perioperative care is discussed. The interaction of the anesthetic agents that target the NMDA receptor and its potential impact on anesthetic care are reviewed [5].

\section{Case Report}

Institutional Review Board approval is not required for retrospective review, and publication of a single patient case report is not required by the Institutional Review Board at Nationwide Children's Hospital (Columbus, Ohio). At the time of the procedure, the patient was an 8-year-old, $28 \mathrm{~kg}$ male, scheduled for an MRI of the brain due to fatigue, altered mental status, abnormal shaking movements, and seizure-like activity along with fever and vomiting. His current medical history included seizures, chronic pain, and anxiety, along with a recent diagnosis of anti-NMDA receptor encephalitis with confirmation by the identification of antibodies in the CSF against the NMDA receptor. The patient had been previously treated with immunosuppressive therapy including corticosteroids, intravenous immunoglobulin, and rituximab. There was no past surgical history. The patient had no known drug allergies. Current 
medications included cyproheptadine and oral nystatin.

General physical examination revealed a normal thyromental distance and a Mallampati Class I airway. The physical examination and preoperative vital signs were unremarkable. The anesthetic plan, risks, benefits and alternatives to general anesthesia were discussed with the parent and informed consent for general anesthesia was obtained. The patient was held nil per os for $8 \mathrm{~h}$, was transported to the MRI suite, and standard American Society of Anesthesiologists' (ASA) monitors were placed. Anesthesia included the administration of a bolus dose of propofol $(1 \mathrm{mg} / \mathrm{kg})$ through an indwelling peripheral intravenous access followed by a propofol infusion at $150 \mu \mathrm{g} /$ $\mathrm{kg} / \mathrm{min}$. Additional medications included ondansetron (3 mg). Intraoperative fluids included $200 \mathrm{~mL}$ of lactated ringers. The MRI, which lasted approximately $60 \mathrm{~min}$, was completed with minimal blood loss and no intraoperative complications. Following completion of the MRI, the patient was transported to the post-anesthesia care unit (PACU). His postoperative course was unremarkable. He was discharged to the inpatient ward from the PACU.

\section{Discussion}

The NMDA receptor is a ligand-gated cation channel responsible for synaptic transmission and plasticity. In anti-NMDA receptor encephalitis, there are antibodies against the NR1NR2 heteromers of the NMDA receptor, which bind glycine and glutamate, respectively $[2,6]$. The auto-immune process leads to accelerated destruction of the NMDA receptor and a decrease in their surface density. This ultimately leads to decreased synaptic NMDA-R-mediated currents and decreased glutamatergic synaptic function $[7,8]$. This reversible loss of the current and synaptic dysfunction is the pathogenesis of the central nervous system effects including memory loss, behavior irregularity, psychosis, and cardiorespiratory changes including arrhythmias and hypoventilation.

As with any anesthetic care, appropriate preoperative preparation begins with a thorough preoperative examination and identification of end-organ effects of the disease process. Of primary concern is the potential for cardiovascular and respiratory involvement. Cardiovascular involvement may include cardiac dysrhythmias (most commonly tachycardia) and blood pressure instability (most commonly hypertension), as a result of autonomic dysfunction [9, 10]. These episodes have been labeled "paroxysmal sympathetic hyperactivity or PSH" [5]. Autonomic dysfunction may also manifest as abrupt episodes of bradycardia or asystole and hypotension. Invasive hemodynamic monitoring may be indicated for patients who are manifesting these clinical signs. Postoperative hemodynamic and continuous electrocardiogram (ECG) monitoring may be indicated based on the intraoperative course. Medications to treat hemodynamic instability and PSH should be readily available including beta adrenergic antagonists, antihypertensive agents, anticholinergic agents, epinephrine, and vasopressors (phenylephrine).

Also of concern is the significant central nervous system (CNS) involvement including psychosis, agitation, memory deficits, seizures, and language disintegration. This may limit patient cooperation and mandate sedation or general anesthesia for non-invasive procedures including radiologic imaging as was required in our patient. Preoperative management strategies to limit the potential for perioperative seizures include optimizing and confirming therapeutic anticonvulsant levels prior to the surgical procedure. Routine anticonvulsant medications should be administered the morning of the procedure despite concerns of the patient's nil per os status with subsequent intraoperative dosing as needed [11]. Alternative routes of delivery (intravenous or rectal) may be required when enteral administration is not feasible. Consultation with the neurology or pharmacology service may be helpful to determine dosing conversion from enteral to intravenous administration or to guide intraoperative redosing. Another intraoperative issue is the potential for intraoperative hyperthermia related to the autonomic instability of anti-NMDA receptor encephalitis. During general anesthesia, blunting of the normal thermoregulatory mechanisms and peripheral vasodilatation with increased heat loss place pediatric patients at a greater risk of hypothermia than adult patients [12]. This concern generally holds true even during MR imaging when radiofrequency radiation emitted by MRI scanners can be absorbed by the patient as heat energy, creating an increase in body temperature [12]. However, given the potential for alterations in body temperature (both hypothermia and hyperthermia), intraoperative temperature monitoring, in accordance with ASA guidelines, is suggested [13]. Significant intraoperative hyperthermia may require investigation and differentiation from more serious conditions such as malignant hyperthermia [14].

CNS involvement may also impact upper airway control and the patient's ability to clear upper airway secretions thereby placing these patients at risk for aspiration. Based on the patient's CNS involvement and nil per os status, techniques to limit the risk of aspiration during anesthetic induction including rapid sequence intubation, and airway control with endotracheal intubation may be indicated $[15,16]$. Given the limited upper airway involvement of our patient, we chose to provide deep sedation with a propofol infusion without airway instrumentation for MR imaging.

Postoperative respiratory failure related to upper airway control, altered central control of ventilation, and impaired clearance of pulmonary secretions may also occur, leading to postoperative respiratory insufficiency. These problems may be exacerbated by the residual effects of anesthetic agents, the surgical procedure, poor cough effort, and the use of postoperative opioids for pain management. These effects may be minimized by the use of short acting agents for sedation and general anesthesia such as propofol, desflurane or remifentanil. As the patient's cognitive state allows, incentive spirometry may improve postoperative respiratory function. Non-invasive techniques of respiratory support such as bilevel positive airway pressure (BiPAP) may be used to facilitate postoperative tracheal extubation [17]. Depending on the severity of the patient's end-organ involvement, postoperative monitoring of respiratory function may be required [18].

The NMDA receptor, along with the gamma-aminobutyric acid (GABA) receptors are the two main receptors postulated to be involved with the mechanisms of general anesthesia [19, 
Table 1. Medications With Direct and Indirect Effects on the NMDA Pathway

\begin{tabular}{l}
\hline Dextromethorphan \\
Ketamine \\
Nitrous oxide \\
Methadone \\
Phencyclidine \\
Magnesium \\
Xenon \\
Pentobarbital \\
Propofol \\
Tramadol \\
\hline
\end{tabular}

20]. Given the involvement of the NMDA pathway, avoidance of medications that act directly through these receptors is suggested (Table 1) [5]. Ketamine binds to the phencyclidine binding site of the NMDA receptor and inhibits glutamate triggered calcium influx. The mechanism of action of ketamine is similar to the pathogenesis of anti-NMDA receptor encephalitis thereby explaining the similarity of ketamine's clinical features with the clinical features of the disease itself [21, 22]. Volatile anesthetic agents such as isoflurane, sevoflurane, and desflurane result in a 50\% depression of NMDA receptormediated currents when used at levels close to the minimum alveolar anesthetic concentration. Although these agents enhance transmission through the GABA transmission, they also inhibit both NMDA-gated currents and NMDA-induced mitochondrial membrane depolarization. Similar effects are noted with propofol thereby leading to indirect effects on the NMDA system [23, 24].

To date, there are a limited number of reports regarding the anesthetic care of patients with anti-NMDA receptor encephalitis [5, 25-27]. The majority of these reports have noted the safe and effective use of the general anesthetic agents including propofol and desflurane, neuromuscular blocking agents including vecuronium and rocuronium, and opioids including fentanyl, remifentanil, and hydromorphone. However, Lapebie et al noted the anecdotal and temporal association of sevoflurane and propofol with worsening of the neurologic status including seizures in a 24-year-old woman with antiNMDA receptor encephalitis related to an ovarian teratoma [25]. Anecdotal experiences by two separate groups of investigators have suggested the potential for an exaggerated effect of propofol on blood pressure with hypotension in these patients $[4,28]$. It has been suggested that there may be decreased anesthetic requirements related to the effects of the disease process on the NMDA system. Other factors that may impact perioperative care include the treatment paradigms including use of immunosuppressive agents, long-term administration of corticosteroids, and plasmapheresis. We would suggest avoidance of agents that have significant and direct effects on the NMDA system (Table 1). In severe cases, the use of benzodiazepines and opioids which have no known effects on the NMDA pathway may be preferred. Additionally, dexmedetomidine with its novel mechanism of action may offer an additional option for sedation during MR imaging, although its potential to cause hemodynamic effects including bradycardia must be considered [29-31].

\section{Acknowledgments}

None to declare.

\section{Financial Disclosure}

There are no financial conflicts of interest.

\section{Conflict of Interest}

The authors have no conflicts of interest to report.

\section{Informed Consent}

Institutional Review Board approval is not required for the retrospective review, and publication of a single patient case report is not required by the Institutional Review Board at $\mathrm{Na}$ tionwide Children's Hospital.

\section{Author Contributions}

A.K. designed and wrote the initial manuscript; M.H. wrote and revised the case report; J.U. and J.T. conceived of the case report, participated in its design and coordination, and helped to draft the final manuscript. All authors read and approved the final manuscript.

\section{References}

1. Vitaliani R, Mason W, Ances B, Zwerdling T, Jiang Z, Dalmau J. Paraneoplastic encephalitis, psychiatric symptoms, and hypoventilation in ovarian teratoma. Ann Neurol. 2005;58(4):594-604.

2. Dalmau J, Tuzun E, Wu HY, Masjuan J, Rossi JE, Voloschin A, Baehring JM, et al. Paraneoplastic anti-Nmethyl-D-aspartate receptor encephalitis associated with ovarian teratoma. Ann Neurol. 2007;61(1):25-36.

3. Granerod J, Ambrose HE, Davies NW, Clewley JP, Walsh AL, Morgan D, Cunningham R, et al. Causes of encephalitis and differences in their clinical presentations in England: a multicentre, population-based prospective study. Lancet Infect Dis. 2010;10(12):835-844.

4. Dalmau J, Lancaster E, Martinez-Hernandez E, Rosenfeld MR, Balice-Gordon R. Clinical experience and laboratory investigations in patients with anti-NMDAR encephalitis. Lancet Neurol. 2011;10(1):63-74.

5. Pryzbylkowski PG, Dunkman WJ, Liu R, Chen L. 
Case report: Anti-N-methyl-D-aspartate receptor encephalitis and its anesthetic implications. Anesth Analg. 2011;113(5):1188-1191.

6. Lynch DR, Anegawa NJ, Verdoorn T, Pritchett DB. Nmethyl-D-aspartate receptors: different subunit requirements for binding of glutamate antagonists, glycine antagonists, and channel-blocking agents. Mol Pharmacol. 1994;45(3):540-545.

7. Manto M, Dalmau J, Didelot A, Rogemond V, Honnorat J. In vivo effects of antibodies from patients with antiNMDA receptor encephalitis: further evidence of synaptic glutamatergic dysfunction. Orphanet J Rare Dis. 2010;5:31.

8. Hughes EG, Peng X, Gleichman AJ, Lai M, Zhou L, Tsou R, Parsons TD, et al. Cellular and synaptic mechanisms of anti-NMDA receptor encephalitis. J Neurosci. 2010;30(17):5866-5875.

9. Dalmau J, Gleichman AJ, Hughes EG, Rossi JE, Peng X, Lai M, Dessain SK, et al. Anti-NMDA-receptor encephalitis: case series and analysis of the effects of antibodies. Lancet Neurol. 2008;7(12):1091-1098.

10. Florance NR, Davis RL, Lam C, Szperka C, Zhou L, Ahmad S, Campen CJ, et al. Anti-N-methyl-D-aspartate receptor (NMDAR) encephalitis in children and adolescents. Ann Neurol. 2009;66(1):11-18.

11. Jones CT, Raman VT, DeVries S, Cole JW, Kelleher KJ, Tobias JD. Optimizing anticonvulsant administration for children before anesthesia: a quality improvement project. Pediatr Neurol. 2014;51(5):632-640.

12. Schneiderbanger D, Johannsen S, Roewer N, Schuster F. Management of malignant hyperthermia: diagnosis and treatment. Ther Clin Risk Manag. 2014;10:355-362.

13. Lo C, Ormond G, McDougall R, Sheppard SJ, Davidson AJ. Effect of magnetic resonance imaging on core body temperature in anaesthetised children. Anaesth Intensive Care. 2014;42(3):333-339.

14. Practice advisory on anesthetic care for magnetic resonance imaging: a report by the Society of Anesthesiologists Task Force on Anesthetic Care for Magnetic Resonance Imaging. Anesthesiology. 2009;110(3):459-479.

15. Tobias JD. Rapid sequence intubation: What does it mean? Does it really matter? Saudi J Anaesth. 2014;8(2):153154.

16. Benington S, Severn A. Preventing aspiration and regurgitation. Anaesth Intensive Care Medicine. 2007;8:368372.

17. Pelosi P, Jaber S. Non-invasive respiratory support in the perioperative period. Curr Opin Anaesthesiol. 2010;3:2233-2228.

18. Lam T, Nagappa M, Wong J, Singh M, Wong D, Chung
F. Continuous pulse oximetry and capnography monitoring for postoperative respiratory depression and adverse events: a systematic review and meta-analysis. Anesth Analg. 2017;125(6):2019-2029.

19. Chau PL. New insights into the molecular mechanisms of general anaesthetics. Br J Pharmacol. 2010;161(2):288307.

20. Dilger JP. The effects of general anaesthetics on ligandgated ion channels. Br J Anaesth. 2002;89(1):41-51.

21. Chapman MR, Vause HE. Anti-NMDA receptor encephalitis: diagnosis, psychiatric presentation, and treatment. Am J Psychiatry. 2011;168(3):245-251.

22. Weiner AL, Vieira L, McKay CA, Bayer MJ. Ketamine abusers presenting to the emergency department: a case series. J Emerg Med. 2000;18(4):447-451.

23. Kingston S, Mao L, Yang L, Arora A, Fibuch EE, Wang JQ. Propofol inhibits phosphorylation of N-methyl-Daspartate receptor NR1 subunits in neurons. Anesthesiology. 2006;104(4):763-769.

24. Orser BA, Bertlik M, Wang LY, MacDonald JF. Inhibition by propofol (2,6 di-isopropylphenol) of the N-methyl-Daspartate subtype of glutamate receptor in cultured hippocampal neurones. Br J Pharmacol. 1995;116(2):17611768.

25. Lapebie FX, Kennel C, Magy L, Projetti F, Honnorat J, Pichon N, Vignon P, et al. Potential side effect of propofol and sevoflurane for anesthesia of anti-NMDA-R encephalitis. BMC Anesthesiol. 2014;14:5.

26. Pascual-Ramirez J, Munoz-Torrero JJ, Bacci L, Trujillo SG, Garcia-Serrano N. Anesthetic management of ovarian teratoma excision associated with anti-N-methyl-Daspartate receptor encephalitis. Int J Gynaecol Obstet. 2011;115(3):291-292.

27. Kawano H, Hamaguchi E, Kawahito S, Tsutsumi YM, Tanaka K, Kitahata H, Oshita S. Anaesthesia for a patient with paraneoplastic limbic encephalitis with ovarian teratoma: relationship to anti-N-methyl-D-aspartate receptor antibodies. Anaesthesia. 2011;66(6):515-518.

28. Splinter WM, Eipe N. Anti-NMDA receptor antibodies encephalitis. Paediatr Anaesth. 2009;19(9):911-913.

29. Tobias JD. Dexmedetomidine: applications in pediatric critical care and pediatric anesthesiology. Pediatr Crit Care Med. 2007;8(2):115-131.

30. Mason KP, Zurakowski D, Zgleszewski SE, Robson CD, Carrier M, Hickey PR, Dinardo JA. High dose dexmedetomidine as the sole sedative for pediatric MRI. Paediatr Anaesth. 2008;18(5):403-411.

31. Wong J, Steil GM, Curtis M, Papas A, Zurakowski D, Mason KP. Cardiovascular effects of dexmedetomidine sedation in children. Anesth Analg. 2012;114(1):193-199. 